\title{
Frequency dependent heat capacity within a kinetic model of glassy dynamics
}

\author{
Dwaipayan Chakrabarti and Biman Bagchi ${ }^{\mathrm{a}}$ \\ Solid State and Structural Chemistry Unit, Indian Institute of Science, Bangalore 560012, India
}

\begin{abstract}
There has been renewed interest in the frequency dependent specific heat of supercooled liquids in recent years with computer simulation studies exploring the whole frequency range of relaxation. The simulation studies can thus supplement the existing experimental results to provide an insight into the energy landscape dynamics. We here investigate a kinetic model of cooperative dynamics within the landscape paradigm for the dynamic heat capacity $C(\omega, T)$ behavior. In this picture, the $\beta$-process is modeled as a thermally activated event in a two-level system and the $\alpha$-process is described as a $\beta$-relaxation mediated cooperative transition in a double well. The model resembles a landscape picture, apparently first conceived by Stillinger [Science 267, 1935 (1995)], where an $\alpha$-process is assumed to involve a concerted series of $\beta$-processes. The model provides a description of the activated hopping in the energy landscape in close relation with the cooperative nature of the hopping event. For suitable choice of parameters, the model predicts a frequency dependent heat capacity that reflects the two-step relaxation behavior. The separation between the two peaks grows as the temperature drops, indicating the stringent constraint on the $\alpha$-process due to the cooperativity requirement. The temperature dependence of the position of the low-frequency peak, due to the $\alpha$-relaxation, shows a non-Arrhenius behavior as observed experimentally. The shape of the $\alpha$-peak is, however, found to be temperature independent. The high-frequency peak appears with considerably larger amplitude than the $\alpha$-peak. We attempt a plausible reason for this observation that is in contrast with the general feature revealed by the dielectric spectroscopy. The relative amplitudes of the $\beta$ - and $\alpha$-peaks in the present framework are found to depend on several characteristic features of the energy landscape, including the extent of cooperativity requirement for the $\alpha$-relaxation and the asymmetry of the double well.
\end{abstract}

\section{INTRODUCTION}

Understanding the complex relaxation phenomena in supercooled liquids has motivated much scientific efforts over decades. ${ }^{1-3}$ The measurement of frequency dependent specific heat, pioneered independently by Birge and Nagel ${ }^{4}$ and by Christensen ${ }^{5}$ in the year 1985 , opened up another approach to this goal. Zwanzig subsequently showed on the basis of linearized hydrodynamics that $c_{p}(\omega)$, the frequency dependent specific heat at constant pressure, could be directly related to the frequency dependent longitudinal viscosity $\eta_{l}(\omega){ }^{6}$ The frequency dependent specific heat has, however, continued to get explored from both experimental ${ }^{7-9}$ and theoretical ${ }^{10-14}$ perspectives in anticipation that specific heat spectroscopy would provide an insight into the energy landscape dynamics.

In practice, one measures the frequency dependent specific heat in the linear response regime following an arbitrary small thermal perturbation that takes the system slightly away from the equilibrium. ${ }^{4} c_{p}(\omega)$ is a linear susceptibility describing the response of the system to this perturbation. One can, however, calculate the frequency dependent specific heat in terms of equilibrium fluctuation of energy fol-

\footnotetext{
a) Author to whom correspondence should be addressed. Electronic mail: bbagchi@sscu.iisc.ernet.in
}

lowing a relevant fluctuation-dissipation theorem derived explicitly by Nielsen and Dyre for a system whose dynamics is described by a master equation. ${ }^{12}$ In this work, we do so for a kinetic model of glassy dynamics that invokes the concept $\beta$-organized- $\alpha$-process ${ }^{15}$ within the landscape paradigm.

The measurements of frequency dependent specific heat currently suffer from a limitation that frequency range up to $10^{4} \mathrm{~Hz}$ can only be probed with the presently available experimental setup. Therefore, the experimentally obtained frequency spectra capture only the $\alpha$-relaxation regime of supercooled liquids. ${ }^{14}$ However, Sceidler et al. ${ }^{14}$ have recently carried out a computer simulation study of a system that models amorphous silica, where they could scan the whole frequency range of interest, revealing the two-peak structure with a notably dominant high-frequency peak. The highfrequency peak shows only a weak temperature dependence and has been ascribed to the vibrational excitations of the system. In their work, ${ }^{14}$ they have applied the Mori-Zwanzig projection operator formalism and made use of an exact transformation formula, due to Lebowitz et al. ${ }^{16}$ to derive a relation between the frequency dependent specific heat $c_{v}(\omega)$ and the autocorrelation function of the temperature fluctuations in the microcanonical ensemble. This relationship, which is identical to the one derived independently by Nielsen ${ }^{13}$ in terms of a fluctuation-dissipation theorem, has 


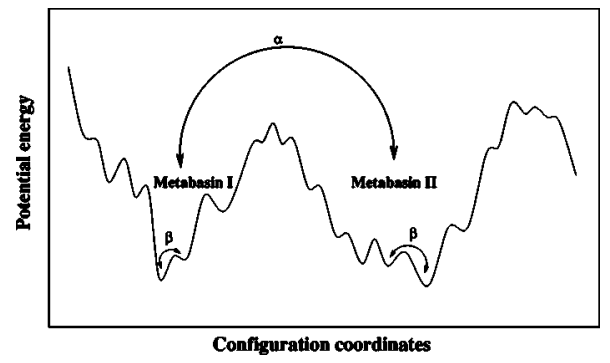

FIG. 1. A schematic representation of the potential energy landscape showing motions within and between metabasins.

allowed the determination of $c_{v}(\omega)$ from computer simulations in equilibrium. A mode-coupling theory (MCT) based calculation has also shown the two-step relaxation behavior in the predicted frequency spectrum of the specific heat. ${ }^{17}$ The dominance of the high-frequency peak is, however, not evident in this work.

The measurements of frequency dependent specific heat in specific heat spectroscopy supplemented by computer simulation studies may prove to be useful in providing insight into the landscape dynamics of supercooled liquids. The landscape paradigm ${ }^{18-21}$ has been widely used to elucidate dynamics of liquids in the supercooled regime. This framework involves the division of the multidimensional configuration space into so called metabasins on the basis of a transition free-energy criterion. Two vastly different time scales thus get entailed, the smaller one due to motions within the metabasins and the longer one due to exchange between the metabasins involving much larger free-energy of activation. In particular, the $\beta$-processes are visualized to originate from activated dynamics within a metabasin, while escape from one metabasin to another is taken to describe an $\alpha$-process. ${ }^{20}$ See Fig. 1 for a schematic representation of the two processes. It is important to recognize that such a description of $\beta$-process within the landscape paradigm corresponds to what is known as the Johari-Goldstein relaxation or "slow" $\beta$-process that is believed to involve local rearrangements. ${ }^{19,20,22}$ This $\beta$-process is so called to distinguish it from an even faster $\beta$-process that is predicted by the mode-coupling theory. ${ }^{23-25}$ Note that the breakdown of the MCT is ascribed to the dominance of relaxation by the thermally activated hopping events, ${ }^{26,27}$ which are unaccounted for in the ideal version of MCT. Recent computer simulation studies $^{28-31}$ have further revealed that hopping is a highly cooperative phenomenon promoted by many body fluctuations; hopping of a tagged particle is often preceded by somewhat larger than normal, but still small amplitude motion of several of its neighbors. ${ }^{31}$ A rather different stringlike cooperative motion has also been found to occur in a model glassforming liquid. ${ }^{32}$

In the present work, we employ a kinetic model of glassy dynamics that attempts to provide a description of the activated hopping within the landscape paradigm in close connection with the cooperative nature of the hopping event. We follow a procedure, as outlined by Nielsen and Dyre, ${ }^{12}$ to compute the frequency dependent heat capacity $C(\omega, T)$ for our model system. For suitable choice of parameters, the

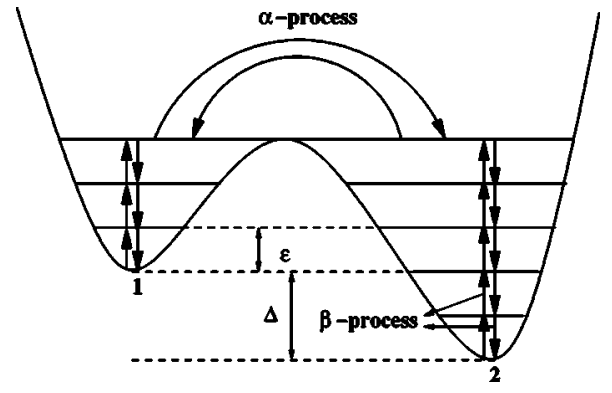

FIG. 2. A schematic representation of the model under consideration. The horizontal lines within a well represent different excitation levels. Note that the energy levels are in general degenerate, as they correspond to the sum of the energies of individual TLSs in the collection.

model predicts a frequency dependent heat capacity that captures many of the features of the two-step relaxation behavior in supercooled liquids. Some of the predictions of our model are in good qualitative agreement with the available experimental and computer simulation results.

The outline of the paper is as follows: In the next section we describe the model. Section III provides the theoretical treatment. We present the results along with discussion in Sec. IV. Section V concludes with a summary of the results and a few comments.

\section{DESCRIPTION OF MODEL}

We model a $\beta$-process as an activated event in a twolevel system (TLS). We label the ground level of a TLS as 0 and the excited level as 1 . The waiting time before a transition can occur from the level $i(=0,1)$ is assumed to be random and is given by the Poissonian probability density function:

$$
\psi_{i}(t)=\frac{1}{\tau_{i}} \exp \left(-t / \tau_{i}\right), \quad i=0,1,
$$

where $\tau_{i}$ is the average time of stay at the level $i$. If $p_{i}(T)$ denotes the canonical equilibrium probability of the level $i$ of a TLS being occupied at temperature $T$, the equilibrium constant $K(T)$ for the population in two levels at temperature $T$ is given by the following relation that obeys the detailed balance:

$$
K(T)=\frac{p_{1}(T)}{p_{0}(T)}=\frac{\tau_{1}(T)}{\tau_{0}(T)}=\exp \left[-\epsilon /\left(k_{B} T\right)\right],
$$

where $\epsilon$ is the energy separation between the two levels in a TLS, and $k_{B}$ is the Boltzmann constant. Here the level 0 is taken to have a zero energy.

Within the framework of the present model, a metabasin is characterized by an $N_{\beta}$ number of such noninteracting two-level systems (TLSs). A given minimum number among the total number $N_{\beta}$ of TLSs must simultaneously be in the excited levels for the occurrence of an $\alpha$-process. We here concentrate on two adjacent metabasins, which we label as 1 and 2 and together call a double well. Figure 2 shows a schematic diagram of two adjacent metabasins with illustration of dynamics within and between them. The respective numbers of TLSs that comprise the metabasins are $N_{\beta}^{(1)}$ and 
$N_{\beta}^{(2)}$. For a collection of $N_{\beta}^{(i)}(i=1,2)$ TLSs, a variable $\zeta_{j}^{i}(t)$, $\left(j=1,2, \ldots, N_{\beta}^{(i)}\right)$ is defined, which takes on a value 0 if at the given instant of time $t$ the level 0 of the TLS $j$ is occupied and 1 if otherwise. $\zeta_{j}^{i}(t)$ is thus an occupation variable. The collective variables $Q_{i}(t)(i=1,2)$ are then defined as

$$
Q_{i}(t)=\sum_{j=1}^{N_{\beta}^{(i)}} \zeta_{j}^{i}(t) .
$$

$Q_{i}(t)$ is therefore a stochastic variable in the discrete integer space $\left[0, N_{\beta}^{(i)}\right] . Q_{i}(t)$ serves as an order parameter for dynamical change involving metabasin $i$. Here an $\alpha$-process is assumed to occur only when all the $\beta$-processes (TLSS) in a metabasin are simultaneously excited, i.e., when $Q_{i}=N_{\beta}^{(i)}$. There is a finite rate of transition $k$ from each of the metabasins when this condition is satisfied. Within the general framework of the model, the double well becomes asymmetric when $N_{\beta}^{(1)} \neq N_{\beta}^{(2)}$, as shown in Fig. 2.

It is worthwhile to note the correspondence of the present description with real physical processes occurring in glassformers. The $\alpha$-process may correspond to large-scale hopping of a particle. For this hopping to occur, however, many small reorientations/rearrangements/displacements are required simultaneously among its neighbors. The activated dynamics within a TLS may well represent small rotations. ${ }^{10}$ In the case of polymer melts which exhibit glassy behavior, the $\beta$-relaxation may involve the motion of side chains. This picture apparently differs from the one drawn by Dyre, ${ }^{33}$ who has argued that large-angle rotations are "causes" and small-angle rotations are "effects." The present picture, however, contains Dyre's one in the sense that small-angle rotations indeed occur following a large-scale jump motion for the completion of relaxation as evident in Fig. 2. The present model is built on a rather symmetrical picture that also necessitates small-angle rotations for a large-angle rotation to occur.

\section{THEORETICAL TREATMENT}

From a theoretical point of view, the treatment of frequency dependent heat capacity can be carried out by employing the linear response assumption. Following Nielsen and Dyre, ${ }^{12}$ the frequency dependent heat capacity $C(\omega, T)$ of our system at temperature $T$ can be given by

$$
C(\omega, T)=\frac{\left\langle E^{2}(T)\right\rangle}{k_{B} T^{2}}-\frac{s}{k_{B} T^{2}} \int_{0}^{\infty} d t e^{-s t}\langle E(t, T) E(0, T)\rangle,
$$

where $s=i \omega, \omega$ being the frequency of the small oscillating perturbation, $i=\sqrt{-1}$, and the angular brackets denote an equilibrium ensemble averaging. $E(t, T)$ stands for the total energy of the system at time $t$ and temperature $T$ and is given by

$$
\begin{aligned}
E(t, T)= & \sum_{n=0}^{N_{\beta}^{(1)}} P_{1}(n ; t, T)\left(N_{\beta}^{(2)}-N_{\beta}^{(1)}+n\right) \epsilon \\
& +\sum_{n=0}^{N_{\beta}^{(2)}} P_{2}(n ; t, T) n \epsilon .
\end{aligned}
$$

Here the lowest level of the well 2 is taken to have zero energy and $P_{i}(n ; t, T)$ denotes the probability that the stochastic variable $Q_{i}$ takes on a value $n$ in the $i$ th well at time $t$ and temperature $T$. The evolution of these probabilities obeys the master equation ${ }^{34}$

$$
\begin{aligned}
\frac{\partial P_{i}(n ; t, T)}{\partial t}= & {\left[\left(N_{\beta}^{(i)}-n+1\right) / \tau_{0}(T)\right] P_{i}(n-1 ; t, T) } \\
& +\left[(n+1) / \tau_{1}(T)\right] P_{i}(n+1 ; t, T) \\
& -\left[\left(N_{\beta}^{(i)}-n\right) / \tau_{0}(T)\right] P_{i}(n ; t, T) \\
& -\left(n / \tau_{1}(T)\right) P_{i}(n ; t, T)-k \delta_{n, N_{\beta}^{(i)}} P_{i}(n ; t, T) \\
& +k \delta_{n, N_{\beta}^{(i \pm 1)}} \delta_{j, i \pm 1} P_{j}(n ; t, T),
\end{aligned}
$$

where the "+" and "-" signs in the indices of the Kronecker delta are for $i=1$ and 2, respectively.

One can have the following compact representation of the set of equations given by Eq. (6) for all possible $n$ and $i$ values

$$
\frac{\partial \mathbf{P}(t, T)}{\partial t}=\mathbf{A}(T) \mathbf{P}(t, T),
$$

where $P_{1}(n ; t, T)$ for $n=0,1, \ldots, N_{\beta}^{(1)}$ and $P_{2}(n ; t, T)$ for $n$ $=0,1, \ldots, N_{\beta}^{(2)}$ together comprise the elements of the column vector $\mathbf{P}(t, T)$ and $\mathbf{A}$ is the transition matrix of order $N$ $=N_{\beta}^{(1)}+N_{\beta}^{(2)}+2$. If $G_{T}(i, t \mid j, 0)$ be the Green's function that gives the probability to be in the state $i$ at a later time $t$ given that the system is in the state $j$ at time $t^{\prime}=0$, the temperature being kept constant at temperature $T$, the matrix of Green's functions also satisfies the rate equation

$$
\frac{d \mathbf{G}_{T}(t)}{d t}=\mathbf{A}(T) \mathbf{G}_{T}(t)
$$

with the initial condition $\mathbf{G}_{T}(0)=\mathbf{I}$, where $\mathbf{I}$ is the identity matrix of order $N$. In terms of Green's functions, one can then rewrite the energy autocorrelation function as

$$
\langle E(t, T) E(0, T)\rangle=\sum_{i=1}^{N} \sum_{j=1}^{N} G_{T}(i, t \mid j, 0) E_{i} E_{j} P_{\mathrm{eq}}(j, T),
$$

where $P_{\text {eq }}(j, T)$ is the equilibrium probability of the state $j$ at $T$. We write $\hat{G}_{T}(i, s \mid j)$ as the Laplace transform of $G_{T}(i, t \mid j, 0)$ :

$$
\hat{G}_{T}(i, s \mid j)=\int_{0}^{\infty} d t e^{-s t} G_{T}(i, t \mid j, 0) .
$$

The frequency dependent heat capacity is then given by

$$
\begin{aligned}
C(\omega, T)= & \frac{\left\langle E^{2}(T)\right\rangle}{k_{B} T^{2}} \\
& -\frac{s}{k_{B} T^{2}} \sum_{i=1}^{N} \sum_{j=1}^{N} \hat{G}_{T}(i, s \mid j) E_{i} E_{j} P_{\mathrm{eq}}(j, T) .
\end{aligned}
$$

The computational procedure involves the numerical evaluation of the Green's functions by an inversion of matrix:

$$
\hat{\mathbf{G}}_{T}(s)=(s \mathbf{I}-\mathbf{A}(T))^{-1},
$$




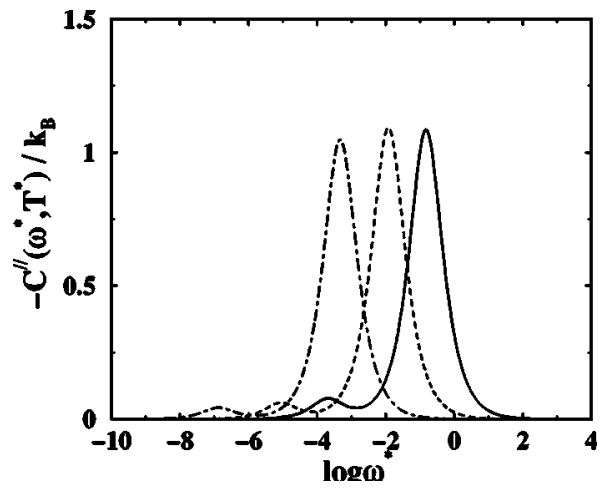

FIG. 3. Frequency dependence of the imaginary part of the dynamic heat capacity $C^{\prime \prime}\left(\omega^{*}, T^{*}\right)$ for our model system with $N_{\beta}^{(1)}=3$ and $N_{\beta}^{(2)}=5$, at three dimensionless reduced temperatures $T^{*}=0.9$ (solid line), $T^{*}=0.8$ (dashed line), and $T^{*}=0.7$ (dotted-dashed line). Temperature $T$ is scaled by the melting temperature $T_{m}$ to have a reduced temperature $T^{*}=T / T_{m}$. Frequency is also scaled by the inverse of $\tau_{1}\left(T_{m}\right)$ to get a dimensionless reduced frequency $\omega^{*}=\omega \tau_{1}\left(T_{m}\right)$. As in the earlier work (Ref. 15) we set $k$

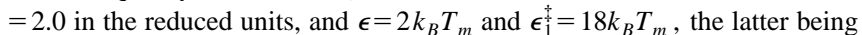
the energy barrier to the transition from the level 1 in a TLS. The presence of a fixed energy barrier to transition from one level to the another within a TLS is expected to impart an Arrhenius temperature dependence of the $\beta$-relaxation within the present framework. The laboratory glass transition temperature $T_{g}$ occurs at a temperature around (2/3) $T_{m}$ (Ref. 1). All temperatures investigated here therefore fall between $T_{m}$ and (2/3) $T_{m}$. The same set of parameter values has been used for all calculations in the present work unless it is specifically mentioned otherwise.

and that of $\mathbf{P}_{\mathrm{eq}}(T)$ from the eigenvector corresponding to the zero eigenvalue of $\mathbf{A}(T)$. In the next section, we present the results with discussion and note the relevance of our results.

The dynamical response of the system in the present framework is expected to be determined by a set of parameters that includes the number $N_{\beta}$ of TLSs in a metabasin, the energy separation $\epsilon$ between the two levels of a TLS, the energy of activation $\epsilon^{\ddagger}$ for barrier crossing within a TLS, the energy asymmetry $\Delta$ between the two adjacent metabasins, and the critical number $N_{c}$ of TLSs required to be in the excited levels at a particular time for the $\alpha$-relaxation to occur. The choice of these parameters has been kept simple in this work though at the expense of being ad hoc at least in some cases. For example, we have taken $N_{c}$ to be equal to $N_{\beta}$ and the value of $\epsilon$ has been taken of the order of $k_{B} T_{m}$, $T_{m}$ being the melting temperature. An approximate estimate of $N_{\beta}$ has been taken from simulation results. ${ }^{30,31}$ The value of the activation energy is a rather difficult guess. We have used the guidance provided by an earlier work of ours. ${ }^{15}$

\section{RESULTS AND DISCUSSION}

In Fig. 3, we show the frequency dependence of the imaginary part of the heat capacity $C^{\prime \prime}\left(\omega^{*}, T^{*}\right)$ calculated for our model system at three different temperatures. The two-peak structure corresponding to the bimodal relaxation behavior as conceived in the model is evident at all temperatures investigated. The peak at high frequencies corresponds to the $\beta$-relaxation and is remarkably dominant. The lowfrequency peak is due to the $\alpha$-relaxation that occurs on a longer time scale. Note that the separation between the position of the $\beta$-peak and that of the $\alpha$-peak grows as tem-

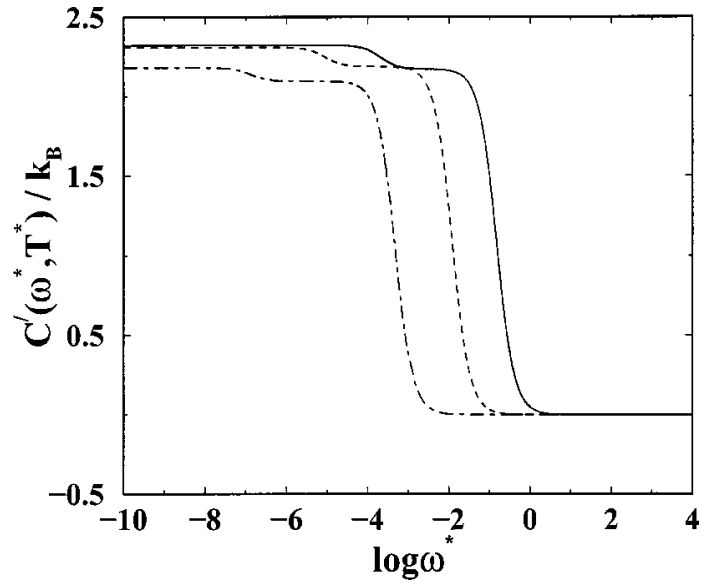

FIG. 4. Frequency dependence of the real part of the dynamic heat capacity $C^{\prime}\left(\omega^{*}, T^{*}\right)$ for our model system at three reduced temperatures $T^{*}=0.9$ (solid line), $T^{*}=0.8$ (dashed line), and $T^{*}=0.7$ (dotted-dashed line).

perature is lowered. This is indicative of the stringent constraint on the $\alpha$-process that arises from the cooperativity requirement.

The features of the two-step relaxation also get reflected in the frequency spectra of the real part of the heat capacity $C^{\prime}\left(\omega^{*}, T^{*}\right)$ as shown in Fig. 4 at three different temperatures. This is expected on the basis of the Kramers-Kronig relation that relates the real and imaginary parts. Whenever $\omega^{-1}$ is on the order of the time scale of a characteristic relaxation process, the system takes up energy inducing an increase in the real part of the heat capacity around that frequency. The dominance of $\beta$-relaxation is again evident from a much larger increase at the high frequencies. The low-frequency limit corresponds to the static heat capacity of the system.

Let us now discuss the temperature dependence of the positions of the peaks as they appear in the frequency spectra of $C^{\prime \prime}\left(\omega^{*}, T^{*}\right)$. The temperature dependence of the $\alpha$-peak position $\omega_{p, \alpha}^{*}\left(T^{*}\right)$ in the reduced scale is shown in Fig. 5 in

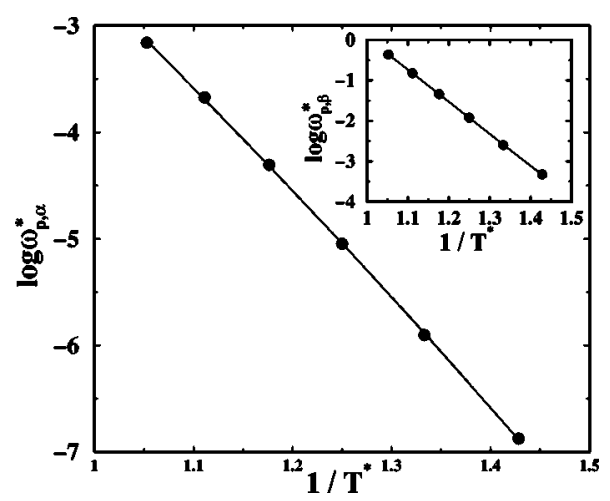

FIG. 5. The $\alpha$-peak frequency $\omega_{p, \alpha}^{*}$, on a logarithmic scale vs the inverse temperature $1 / T^{*}$. The solid line and dashed line correspond to two nearly indistinguishable fits to the data with the Vogel-Fulcher-Tammann equation and a three-parameter scaling law (see text). From the fits, $T_{0, \mathrm{VFT}}$ $=0.154$ and $T_{0, \text { scl }}=0.475$ in the reduced units. The inset shows the temperature dependence of the $\beta$-peak frequency $\omega_{p, \beta}^{*}$ in a $\log \omega_{p, \beta}^{*}$ vs $1 / T^{*}$ plot. The solid line is a linear fit to the data with a slope of 7.912 in the reduced temperature units. 


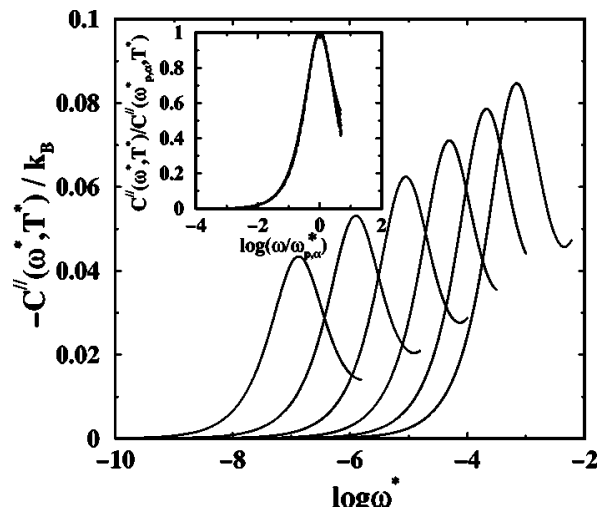

FIG. 6. The frequency spectra of the imaginary part of the dynamic heat capacity $C^{\prime \prime}\left(\omega^{*}, T^{*}\right)$ at different temperatures showing the low-frequency $\alpha$-peak only. From the right to the left $T^{*}=0.95,0.9,0.85,0.8,0.75,0.7$, respectively. The inset shows the same curves, in dotted lines, scaled by the height of the respective peaks vs $\log \left(\omega^{*} / \omega_{p, \alpha}^{*}\right)$. The solid line is a fit to the curve for $T^{*}=0.7$ with a three-parameter equation that is a good frequency domain representation of the time domain Kohlrausch-Williams-Watts stretched exponential form. The dashed line is a fit to the same curve with the Debye response function. The curves along with the two fits are nearly indistinguishable. The details of the fit are given in the text. The range of frequency shown here is restricted at the high frequency side due to the presence of the $\beta$-peak that leads to a break down of the fitting.

a $\log \omega_{p, \alpha}^{*}\left(T^{*}\right) \quad$ vs $1 / T^{*}$ plot. The data fit well to the Vogel-Fulcher-Tammann (VFT) equation: $\omega_{p, \alpha}^{*}\left(T^{*}\right)$ $=\omega_{0, \mathrm{VFT}} \exp \left[-A_{\mathrm{VFT}} /\left(T^{*}-T_{0, \mathrm{VFT}}\right)\right]$. The small curvature of the fitted curve is, however, notable and has been observed experimentally as well. ${ }^{4}$ As in Ref. 4 , we show in Fig. 5 a second fit to the data with a scaling law: $\omega_{p, \alpha}^{*}\left(T^{*}\right)$ $=\omega_{0, \text { scl }}\left[\left(T^{*}-T_{0, \mathrm{scl}}\right) / T_{0, \mathrm{scl}}\right]$. The two fits are nearly indistinguishable. The inset of Fig. 5 shows the temperature dependence of the $\beta$-relaxation peak position $\omega_{p, \beta}^{*}\left(T^{*}\right)$ in a similar plot. The linear fit corresponds to the Arrhenius behavior as expected from the supposition of the model.

Since the experiments till date could probe only the $\alpha$-relaxation regime of the frequency spectrum, we have investigated the features of the $\alpha$-relaxation peak as predicted by our model system in a bit more detail. In Fig. 6, we concentrate on the $\alpha$-relaxation regime of the frequency spectra of $C^{\prime \prime}\left(\omega^{*}, T^{*}\right)$ at different temperatures to get an enlarged view of the $\alpha$-relaxation peak. It is evident that the area under the $\alpha$-peak becomes smaller as temperature drops. This implies that the cooperative component of the configurational part of the heat capacity diminishes with decreasing temperature. See Ref. 14 for a detailed discussion. Although the height and the position of the $\alpha$-peak have been found to change with temperature, its shape seems to show no such dependence. This can be evident if one constructs a master plot by scaling both the height of the peak and the frequency. This is demonstrated in the inset of Fig. 6 where we plot $C^{\prime \prime}\left(\omega^{*}, T^{*}\right) / C^{\prime \prime}\left(\omega_{p, \alpha}^{*}, T^{*}\right)$ vs $\log \left(\omega^{*} / \omega_{p, \alpha}^{*}\right)$ at different temperatures for the frequency range of interest. The curves at different temperatures appear to be superimposed on each other with negligible error. It is therefore reasonable to conclude that the shape of the $\alpha$-peak here does remain independent of temperature. The computer simulation study reported in Ref. 14 makes a similar observation on the shape of the $\alpha$-peak.

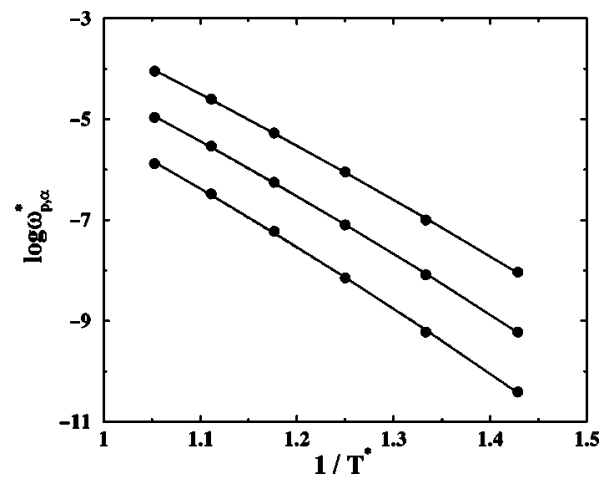

FIG. 7. The $\alpha$-peak frequency $\omega_{p, \alpha}^{*}$, on a logarithmic scale vs the inverse temperature $1 / T^{*}$ for three different sets of $\left\{N_{\beta}^{(1)}, N_{\beta}^{(2)}\right\}$ values. For each set, the solid line and dashed line correspond to two nearly indistinguishable fits to the data with the Vogel-Fulcher-Tammann equation and a threeparameter scaling law. From the fits, $T_{0, \mathrm{VFT}}=0.174$ and $T_{0, \mathrm{scl}}=0.485$ for $\{4,6\}$ (top), $T_{0, \mathrm{VFT}}=0.197$ and $T_{0, \mathrm{scl}}=0.496$ for $\{5,7\}$ (middle), and $T_{0, \mathrm{VFT}}$ $=0.203$ and $T_{0, \mathrm{scl}}=0.498$ for $\{6,8\}$ (bottom).

The results presented here until now have been for a single set of $N_{\beta}^{(1)}, N_{\beta}^{(2)}, \epsilon$ and $\epsilon_{1}^{\ddagger}$ values. It is important to know how the predicted results are dependent on the choice of model parameters, in particular on $N_{\beta}^{(1)}$ and $N_{\beta}^{(2)}$, before one judges the merits of the model. To this end, we have explored the parameter dependence of the predictions of our model. The two-step relaxation behavior has been found to be revealed in the predicted heat capacity spectra of the model for a few other choices of $\left\{N_{\beta}^{(1)}, N_{\beta}^{(2)}\right\}$ as well, specifically, for $\{4,6\},\{5,7\}$, and $\{6,8\}$. For each of these parameter sets, a similar non-Arrhenius behavior of the temperature dependence of the $\alpha$-peak position has been observed as shown in Fig. 7. The Vogel temperature $T_{0, \mathrm{VFT}}$ obtained from the VFT fit shifts to higher values along the series as one would expect from an increasingly stringent cooperativity requirement. Although the magnitude of the shift is small, the trend is quite clear. The shape of the $\alpha$-peak has also been found to remain independent of temperature along the series (data not shown). For $\{6,9\}$, the amplitude of the $\alpha$-peak gets diminished to a large extent by the time $T^{*}$ $=0.7$ is reached, while the two-peak structure does not appear at all even at $T^{*}=0.95$ for $\{6,10\}$. The two-step relaxation behavior also does not get evident from the predicted dynamic heat capacity spectra in the temperature range explored here for even $\{2,6\}$. It follows therefore that a difference of 4 in $N_{\beta}^{(2)}$ and $N_{\beta}^{(1)}$ with the set of $\epsilon$ and $\epsilon_{1}^{*}$ values used here is large enough to suppress any manifestation of the two-step relaxation behavior within the present framework. On considering varying asymmetry of the two adjacent metabasins, we further make an interesting observation as illustrated in Fig. 8. Here, we have varied $N_{\beta}^{(2)}$ with $N_{\beta}^{(1)}, \epsilon$ and $\epsilon_{1}^{\ddagger}$ held fixed. For a symmetric double well, only one peak appears in the frequency spectrum of the imaginary part of the dynamic heat capacity. The $\alpha$-peak disappears as an $\alpha$-process is inconsequential from an energy consideration for the symmetric double well. The amplitude of the $\alpha$-peak is the largest with the least asymmetry and gradually diminishes with growing asymmetry before it gets suppressed completely. The results suggest that the relative amplitudes 


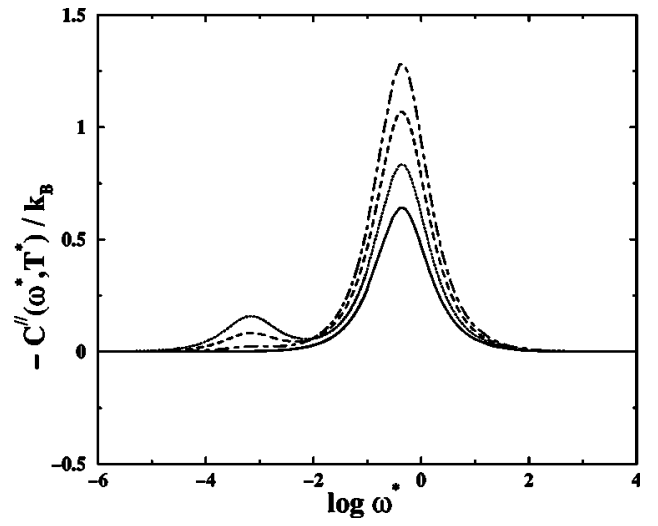

FIG. 8. The frequency spectra of the imaginary part of the dynamic heat capacity $C^{\prime \prime}\left(\omega^{*}, T^{*}\right)$ at temperature $T^{*}=0.95$ for four choices of $N_{\beta}^{(2)}$ with $N_{\beta}^{(1)}$ kept fixed: (a) $\{3,3\}$ (solid line), (b) $\{3,4\}$ (dotted line), (c) $\{3,5\}$ (dashed line), and (d) $\{3,6\}$ (dotted-dashed line).

of the $\alpha$-peak may provide an insight into the energy asymmetry between the metabasins in the energy landscape of the system. It may be noted in this context that if the $\beta$-processes are not associated with any energy cost (i.e., $\epsilon=0$ ) even though being activated events (i.e., with a nonzero energy of activation $\epsilon^{\ddagger}$ for barrier crossing), one would expect the strongly temperature dependent high-frequency peak to disappear.

In the inset of Fig. 6, we have also shown a fit to the curve for $T^{*}=0.7$ with a three-parameter equation ${ }^{35}$ that is a good frequency domain representation of the time domain Kohlrausch-Williams-Watts (KWW) stretched exponential form. From the fit parameters we obtain the stretching parameter $\beta_{\mathrm{KWW}}=0.94$. Such a $\beta_{\mathrm{KWW}}$ value implies very weakly nonexponential behavior. In fact, a fit with the Debye response function that corresponds to a single exponential behavior is found to be reasonable and for some of the other sets of $\left\{N_{\beta}^{(1)}, N_{\beta}^{(2)}\right\}$, it is found to be as good as the one with the three-parameter equation with a stretching parameter very close to unity. This is reasonable as spatially heterogeneous domains, which is believed to be the primary reason for the stretched exponential relaxation in supercooled liquids, ${ }^{36-38}$ has not been considered in the present calculations. The heterogeneous dynamics in different domains can be included either through a distribution of $\epsilon$ (the separation between the energy levels within a TLS) or through a distribution of barrier height for transition from one level to the other within a TLS. When the heterogeneity is included, the exponent $\beta_{\mathrm{KWW}}$ is expected to decrease considerably as indeed found in the treatment of structural relaxation within a similar model where we have considered a distribution of $\epsilon$. ${ }^{39}$

The remarkably dominant $\beta$-peak as predicted by our model merits further consideration. In order to trace back its origin, we note that the constraint of cooperativity on the $\alpha$-relaxation allows the system to take up energy only through the localized $\beta$-processes unless the condition is satisfied. The system takes up more energy through the excitation of individual $\beta$-processes (that collectively bring about the $\alpha$-process) than the $\alpha$-process itself which corresponds to a transition from one metabasin to another. This argument is

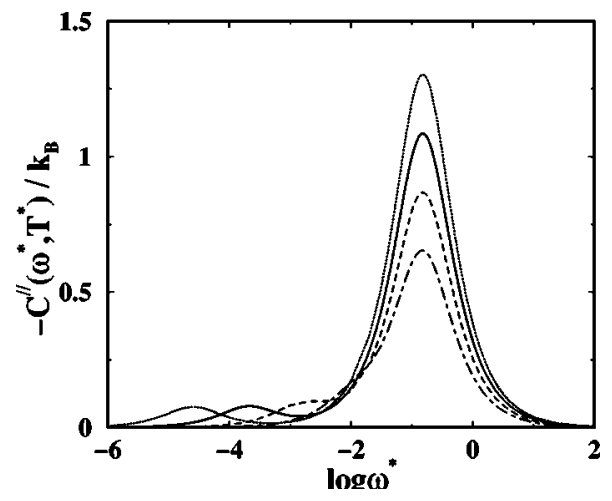

FIG. 9. The frequency spectra of the imaginary part of the dynamic heat capacity $C^{\prime \prime}\left(\omega^{*}, T^{*}\right)$ at temperature $T^{*}=0.9$ for four sets of $\left\{N_{\beta}^{(1)}, N_{\beta}^{(2)}\right\}$ values with $N_{\beta}^{(2)}-N_{\beta}^{(1)}$ held fixed: (a) $\{4,6\}$ (dotted line), (b) $\{3,5\}$ (solid line), (c) $\{2,4\}$ (dotted line), and (d) $\{1,3\}$ (dotted-dashed line).

substantiated in Fig. 9, where we focus on the amplitude of the $\beta$-peak relative to that of the $\alpha$-peak in the frequency spectra of the imaginary part of the dynamic heat capacity. Note that the $\alpha$-peak is not distinctly observed for $\{1,3\}$ as the separation between the two peaks is not enough. A decrease in the requirement of cooperativity for the $\alpha$-process is found to result in a fall in the relative amplitude of the $\beta$-peak. One can, therefore, define a parameter $Q_{h c}^{\alpha \beta}$ as the ratio of the amplitude of the $\beta$-peak to that of the $\alpha$-peak, which is in general temperature dependent. That is, $Q_{h c}^{\alpha \beta}(T)$ $=C^{\prime \prime}\left(\omega_{p, \beta}, T\right) / C^{\prime \prime}\left(\omega_{p, \alpha}, T\right)$. The parameter $Q_{h c}^{\alpha \beta}(T)$ may serve as a measure of the cooperativity needed for $\alpha$-relaxation to take place.

\section{CONCLUSION}

Let us first summarize the present work. We have employed a kinetic model of glassy dynamics that considers cooperativity through the constraint of $\beta$-organized- $\alpha$ process within the landscape paradigm. The two-step relaxation behavior as conceived in the model gets revealed in the frequency dependent heat capacity of the model for a reasonable range of parameter values. The analysis of the predicted dynamic heat capacity spectra suggests the following emergent features of our model: (i) The $\alpha$-peak frequency has a non-Arrhenius temperature dependence. (ii) The shape of the $\alpha$-peak as it appears in the frequency spectrum of the imaginary part of the dynamic heat capacity is invariant in temperature. (iii) The amplitude of the $\beta$-peak is considerably larger than that of the $\alpha$-peak.

While the first (the non-Arrhenius temperature dependence) of the above conforms to a number of experimental results, the second one has been observed in a recent computer simulation study. ${ }^{14}$ The third one, however, does invite a few comments. Although a remarkably dominant highfrequency peak has been reported in Ref. 14, it is rather ascribed to the vibrational excitations of the system and is likely to be more relevant with the "fast" $\beta$-process typically appearing in the mode-coupling theory predictions. The activated dynamics with an Arrhenius-type temperature dependence characterizing the $\beta$-process considered in the present work is on the other hand a typical feature of the Johari- 
Goldstein relaxation or "slow" $\beta$-process. ${ }^{19,20,22}$ A dominant $\beta$-peak with a strong temperature dependence suggests that such a $\beta$-peak should be observable in the specific heat spectroscopy even with its limited frequency range if it is explored at low enough temperatures. We, however, note that the experimentally obtained dynamic heat capacity spectra are yet to capture the two-step relaxation behavior even though dielectric spectroscopy has revealed distinct two peaks for a number of glassformers, and it is rather the $\beta$-peak that is the weaker one. An address to this issue in further detail is beyond the scope of the present work.

The present work suggests that the relative amplitudes of the two peaks may provide insight into the microscopic mechanism of the relaxation processes in deeply supercooled liquids. In view of this, further measurements of frequency dependent specific heat of various glass forming liquids will certainly be worthwhile. A recent simulation study by van Ee et $a{ }^{40}$ of computer model of amorphous $\mathrm{Ni}_{81} \mathrm{~B}_{9}$ has shown that the hopping mode is not only collective but appears to involve rather large number of neighbors. This study seems to support the picture that coherence among neighbors is a prerequisite for large scale hopping. Such a scenario is compatible with our present picture where $\beta$-process may be identified with small amplitude motion while hopping is the jump between the two minima shown in Fig. 2. We further argue here that a predominant $\beta$-peak would be a characteristic feature of Stillinger's picture which assumes an $\alpha$-process to involve a concerted series of $\beta$-processes. ${ }^{22}$

Several other comments on the present work are in order. First, the present model can be taken to belong to the class of kinetically constrained models ${ }^{41}$ that attempts to provide a description of glassy dynamics by imposing dynamical constraints on the allowed transitions between different configurations of the system, while maintaining the detailed balance. In particular, our model resembles the models of hierarchically constrained dynamics of glassy relaxation, due originally to Palmer et al., ${ }^{42}$ in the spirit that brings in cooperativity. Second, the high-frequency peak for real liquids is likely to draw contribution from $\beta$-relaxation as well as vibrational excitations. In some cases, specifically at low temperatures close to the glass transition, it is possible that these two can be sufficiently separated to give rise to an additional peak in the imaginary part of the frequency dependent specific heat. Third, it is imperative to compare the two-peak structure of the frequency spectrum of the imaginary part of the dynamic heat capacity with its dielectric analog. In contrary to the prediction our model makes on dynamic heat capacity behavior, it is rather the predominance of the $\alpha$-peak that has been observed in a vast body of experimental data on dielectric relaxation. While the fluctuation in energy within the present framework of the model that has an energy landscape picture at the backdrop translates easily into the calculation of the frequency dependent heat capacity, the model as such does not allow us to calculate the frequency dependence of the dielectric constant. The latter needs further development of the model. However, the well-known bimodal frequency dependence of the dielectric relaxation in supercooled liquids can be at least qualitatively understood from the present description of $\beta$ - and $\alpha$-processes. We es- sentially follow the description of Lauritzen and Zwanzig ${ }^{43}$ in assuming that a $\beta$-process can be taken to correspond to a two-site angular jump of individual molecules by a small angle around some axis. These individual, uncorrelated angular jumps lead to a partial relaxation of the total electric moment $\mathbf{M}(t)$ of the whole system [note that $\mathbf{M}(t)$ is the sum of the dipole moment of the individual molecules]. The dielectric susceptibility spectrum can be obtained from the auto-time correlation function of $\mathbf{M}(t)$ by using the linear response theory. ${ }^{44}$ Since $\mathbf{M}(t)$ is a sum of a relatively large number of individual dipole moments, the former is a Gaussian Markov process and thus the time correlation function of the $\beta$-relaxation mediated part must decay exponentially. As noted earlier, this $\beta$-relaxation mediated decay is incomplete because all the jumps are small and restricted. Thus, it is fair to assume the following form for the auto-time correlation function of $\mathbf{M}(t)$,

$C_{M}(t)=\left\langle M_{\beta}^{2}\right\rangle \exp \left(-t / \tau_{\beta}\right)+\left(\left\langle M_{0}^{2}\right\rangle-\left\langle M_{\beta}^{2}\right\rangle\right) \exp \left(-t / \tau_{\alpha}\right)$,

where $\tau_{\beta}$ and $\tau_{\alpha}$ are the time scales of $\beta$-relaxation and $\alpha$-relaxation, respectively. In the above equation $\left\langle M_{\beta}^{2}\right\rangle$ is the value by which the mean-square total dipole moment decays due to $\beta$-relaxation alone from the initial value of $\left\langle M_{0}^{2}\right\rangle$. The rest of the decay to zero occurs via the $\alpha$-process. This suggests that with well separated $\tau_{\beta}$ and $\tau_{\alpha}$, one would observe bimodal dispersion. However, the calculation of $\left\langle M_{\beta}^{2}\right\rangle$ would require a more detailed model than the one attempted here.

The present study suggests several future problems. First, it would be interesting to investigate the frequency dependence of the specific heat of several molecular liquids of varying fragility in computer simulations. In some cases, one should be able to discern a three-peak structure. Second, it would be also of interest to investigate if the frequency dependent specific heat can be used to study the so-called Boson peak which has drawn much attention in recent times. ${ }^{45}$ Third, a comparison of the frequency dependence of specific heat with that of the dynamic structure factor of certain model systems would provide us insight to decide on whether the microscopic mechanisms of relaxation vary for different modes. Finally, a generalization of the present model or an altogether different model to describe the frequency dependence of dynamic heat capacity and that of dielectric response in the same framework would be a worthwhile undertaking.

\section{ACKNOWLEDGMENTS}

We thank Professor N. Menon and Professor C. A. Angell for useful discussions. This work was supported in parts by grants from CSIR and DST, India. D.C. acknowledges the University Grants Commission (UGC), India for providing the Research Fellowship.

\footnotetext{
${ }^{1}$ C. A. Angell, K. L. Ngai, G. B. McKenna, P. F. McMillan, and S. W. Martin, J. Appl. Phys. 88, 3113 (2000).

${ }^{2}$ P. G. Debenedetti and F. H. Stillinger, Nature (London) 410, 259 (2001).

${ }^{3}$ U. Mohanty, Adv. Chem. Phys. 89, 89 (1994).

${ }^{4}$ N. O. Birge and S. R. Nagel, Phys. Rev. Lett. 54, 2674 (1985).

${ }^{5}$ T. Christensen, J. Phys. (Paris), Colloq. 46, C8-635 (1985).
} 
${ }^{6}$ R. Zwanzig, J. Chem. Phys. 88, 5831 (1988).

${ }^{7}$ N. O. Birge, Phys. Rev. B 34, 1631 (1986).

${ }^{8}$ P. K. Dixon and S. R. Nagel, Phys. Rev. Lett. 61, 341 (1988)

${ }^{9}$ N. Menon, J. Chem. Phys. 105, 5246 (1996).

${ }^{10}$ D. W. Oxtoby, J. Chem. Phys. 85, 1549 (1986).

${ }^{11}$ J. Jäckle, Physica A 162, 377 (1990).

${ }^{12}$ J. K. Nielsen and J. C. Dyre, Phys. Rev. B 54, 15754 (1996).

${ }^{13}$ J. K. Nielsen, Phys. Rev. E 60, 471 (1999).

${ }^{14}$ P. Sceidler, W. Kob, A. Latz, J. Horbach, and K. Binder, Phys. Rev. B 63, 104204 (2001).

${ }^{15}$ D. Chakrabarti and B. Bagchi, J. Chem. Phys. 120, 11678 (2004).

${ }^{16}$ J. L. Lebowitz, J. K. Percus, and L. Verlet, Phys. Rev. 153, 250 (1967).

${ }^{17}$ U. Harbola and S. P. Das, Phys. Rev. E 64, 046122 (2001).

${ }^{18}$ M. Goldstein, J. Chem. Phys. 51, 3728 (1969).

${ }^{19}$ G. P. Johari and M. Goldstein, J. Chem. Phys. 53, 2372 (1970); 55, 4245 (1971).

${ }^{20}$ F. H. Stillinger, Science 267, 1935 (1995).

${ }^{21}$ S. Sastry, P. G. Debenedetti, and F. H. Stillinger, Nature (London) 393, 554 (1998).

${ }^{22}$ D. J. Wales, Energy Landscapes (Cambridge University Press, Cambridge, 2003).

${ }^{23}$ U. Bengtzelius, W. Götze, and A. Sjölander, J. Phys. C 17, 5915 (1984).

${ }^{24}$ E. Leutheusser, Phys. Rev. A 29, 2765 (1984).

${ }^{25}$ W. Götze and L. Sjögren, Rep. Prog. Phys. 55, 241 (1992).

${ }^{26}$ C. A. Angell, J. Phys. Chem. Solids 49, 863 (1988).

${ }^{27}$ B. Bagchi, J. Chem. Phys. 101, 9946 (1994).

${ }^{28}$ G. Wahnström, Phys. Rev. A 44, 3752 (1991).

${ }^{29}$ H. Miyagawa, Y. Hiwatari, B. Bernu, and J. P. Hansen, J. Chem. Phys. 88, 3879 (1988).
${ }^{30}$ S. Bhattacharyya and B. Bagchi, Phys. Rev. Lett. 89, 025504 (2002).

${ }^{31}$ S. Bhattacharyya, A. Mukherjee, and B. Bagchi, J. Chem. Phys. 117, 2741 (2002).

${ }^{32}$ C. Donati, J. F. Douglas, W. Kob, S. J. Plimpton, P. H. Poole, and S. C. Glotzer, Phys. Rev. Lett. 80, 2338 (1998).

${ }^{33}$ J. C. Dyre, Phys. Rev. E 59, 2458 (1999).

${ }^{34}$ N. G. van Kampen, Stochastic Processes in Physics and Chemistry (Elsevier Science, Amsterdam, 1992).

${ }^{35}$ The three-parameter equation in the frequency domain to fit a $X^{\prime \prime}(\omega)$ vs $\omega$ curve reads as $X^{\prime \prime}(\omega)=X_{p}^{\prime \prime}(\omega) /\left[1-b+b /(1+b)\left\{b\left(\omega_{p} / \omega\right)+\left(\omega / \omega_{p}\right)^{b}\right\}\right]$, where $X_{p}^{\prime \prime}, \omega_{p}$, and $b$ are the peak height, peak position, and the shape parameter, respectively. These give the KWW parameters through definite relationships. In particular, $b \approx \beta_{\mathrm{KWw}}$. See R. Bergman, J. Appl. Phys. 88, 1356 (2000)

${ }^{36}$ M. D. Ediger, Annu. Rev. Phys. Chem. 51, 99 (2000).

${ }^{37}$ H. Sillescu, J. Non-Cryst. Solids 243, 81 (1999).

${ }^{38}$ R. Richert, J. Phys.: Condens. Matter 14, R703 (2002).

${ }^{39}$ D. Chakrabarti and B. Bagchi (unpublished).

${ }^{40}$ L. D. van Ee, B. J. Thijsee, and J. Sietsma, Phys. Rev. E 57, 906 (1998).

${ }^{41}$ F. Ritort and P. Sollich, Adv. Phys. 52, 219 (2003).

${ }^{42}$ R. G. Palmer, D. L. Stein, E. Abrahams, and P. W. Anderson, Phys. Rev. Lett. 53, 958 (1984).

${ }^{43}$ J. I. Lauritzen, Jr. and R. Zwanzig, Adv. Mol. Relax. Processes 5, 339 (1973).

${ }^{44}$ R. Zwanzig, Nonequilibrium Statistical Mechanics (Oxford University Press, New York, 2001).

${ }^{45}$ V. Lubchenko and P. G. Wolynes, Proc. Natl. Acad. Sci. U.S.A. 100, 1515 (2003) 